\title{
The impact of Coronavirus disease 2019 (COVID-19) pandemic on migraine disorder
}

\author{
Faraidoon Haghdoost ${ }^{1} \cdot$ Cheryl Carcel $^{2} \cdot$ Deepak Chandrasekhar $^{3} \cdot$ Anthony Rodgers $^{1} \cdot$ Candice Delcourt $^{1,4}$ (D)
}

Received: 8 March 2021 / Revised: 28 April 2021 / Accepted: 30 April 2021 / Published online: 5 May 2021

(c) Springer-Verlag GmbH Germany, part of Springer Nature 2021

\begin{abstract}
Objective To evaluate the impact of the Coronavirus disease of 2019 (COVID-19) pandemic on patients with migraine. Methods Self-reported data from a migraine tracking smartphone application, Migraine Buddy, were used. Data were collected from users who reported at least one attack in the Jan, Feb, Mar and Apr of 2018, 2019 and 2020. In addition, a survey was conducted to evaluate the impact of COVID-19 on migraine management.

Results On average, data from 124,717 users per month (mean age $36.3 \pm 10.9$ years and $89 \%$ female) were collected. Overall, the mean frequency of migraine headache was higher in 2020 than in 2019 and higher in 2019 than in 2018. The four commonest headache triggers in 2018, 2019 and 2020 were stress in 39.7, 38.4 and 36.1\%, lack of sleep in 25, 25 and 22.8\%, neck pain, 20, 20.4 and 19.3 and anxiety in 19,18.4 and 18.4\% of participants, respectively. 1689 users participated in the survey and they reported that they preferred face-to-face (54.29\%) to telehealth (11.9\%) consultations.

Conclusion An increase in migraine frequency from 2018 to 2020 was reported by the users of the mobile phone. This could reflect a real increase or change in reporting habits. Stress, lack of sleep, neck pain and anxiety were the commonest attack triggers. The frequency of these triggers decreased slightly in 2020 compared to 2019 and 2018. An increase in telehealth consultations with specialists was reported in the survey but migraine patients preferred face-to-face consultations.
\end{abstract}

Keywords Anxiety $\cdot$ COVID-19 $\cdot$ Frequency $\cdot$ Headache $\cdot$ Migraine $\cdot$ Stress $\cdot$ Trigger

Anthony Rodgers and Candice Delcourt Senior authors contributed equally.

Candice Delcourt

cdelcourt@georgeinstitute.org.au

Faraidoon Haghdoost

FHaghdoost@georgeinstitute.org.au

Cheryl Carcel

ccarcel@georgeinstitute.org.au

Deepak Chandrasekhar

deepak@healint.com

Anthony Rodgers

arodgers@georgeinstitute.org

1 The George Institute for Global Health, The University of New South Wales, Sydney, NSW, Australia

2 The George Institute for Global Health, The University of New South Wales, Royal Prince Alfred Hospital, The University of Sydney, Sydney, NSW, Australia

3 Healint Pte Ltd, Singapore, Singapore

4 Department of Clinical Medicine, Faculty of Medicine, Health and Human Sciences, Macquarie University, Macquarie Park, NSW, Australia

\section{Introduction}

As of March the 1st 2021, the COVID-19 pandemic has affected over 110 million people worldwide of which about $1,850,000$ have died and around 60,000,000 have recovered [1]. The impact of the COVID-19 pandemic on health care delivery systems has been overwhelming in many countries. The unexpected number of cases has mobilised hospital teams and resources, with the 'non-essential' care of nonCOVID patients cancelled or postponed, creating a challenge in providing adequate care for patients with chronic illnesses.

Migraine disease is recognised as one of the top five causes of disability worldwide [2]. It is one of the commonest reasons for neurological review. During the pandemic, face-to-face consultations with a regular neurologist have been postponed or converted to telemedicine consultation to ensure physical distancing. This change has left some patients managing their migraine at home with limited medical support [3-5]. 
Using Migraine Buddy data, the most commonly used mobile phone application for migraine and headache tracking, we aim to investigate the impact of the COVID-19 pandemic on patients experiencing migraine.

\section{Methods}

This was a cross-sectional study using data from a free migraine tracking smartphone application, Migraine Buddy. Migraine Buddy is used by more than 2 million users globally and is available in several languages including English, Dutch, French, German, Italian, Japanese, Polish, Portuguese and Spanish. This application is designed to help users track, find migraine patterns, and report headaches and associated triggers, which can be exported to share during physician consultations to diagnose and monitor migraine. In addition, the application has built-in reminders to allow users to input all data points related to migraine attack and can send targeted questionnaires to gain real-time insights into migraine management.

For this analysis, data were extracted from Migraine Buddy for January, February, March, or April of 2018, 2019 and 2020. Self-reported data were collected from the users who had reported at least one migraine attack in the respective months. Demographic characteristics including gender, age and country were collected. Mean headache frequency per month and percentage of the four most reported triggers of headache attacks were collected and reported.

In addition, a survey including five questions to evaluate the impact of COVID-19 pandemic on migraine management from the perspective of patients was sent to Migraine Buddy users who were based in English speaking countries from 16 to 30th of July 2020. The survey questions are reported in the result section.

Summary statistics are reported as mean \pm standard deviation (SD) or number (\%). An online $t$-test calculator was used to compare the mean frequency of headaches between the years [6]. $P$ value less than 0.05 was considered as statistically significant. The SPSS software package was used to prepare the infographic. User data were deidentified and users were given the option to share their data for research purposes during onboarding in the Migraine Buddy application.

\section{Results}

In the current study, data from an average of 124,717 users (minimum 109,529 users in Apr 2018 to maximum 151,724 users in Jan 2020) were collected. Table 1 shows the basic characteristics of the users included in this analysis.
Overall, users were predominantly female (mean 63.1\%), and the mean $( \pm$ SD) age was $36.3 \pm 10.9$ years. Most of the users were from the United States of America (47\%) and the rest were from France (7\%), the United Kingdom (7\%), Canada (5.6\%), Germany (4.3\%), Japan (3.9\%), Spain (3.2\%), Italy (2.6\%), Australia (2.1\%), Switzerland (0.8\%), and other countries $(16.3 \%)$.

Mean headache frequencies per month in studied months are reported in Table 2. In general, headache frequency per month was reported higher in 2020 than 2019 and higher in 2019 than 2018. Mean headache frequency $( \pm$ SD) per month in 2018, 2019 and 2020 were $4.88 \pm 4.96,5.23 \pm 5.28$ and $5.39 \pm 5.36$, respectively. The increase in frequency between years and between the same month of each year was statistically significant $(p<0.001)$, with the exception of the mean headache frequency in Jan 2020 compared with Jan $2019(p=0.052)$.

The four most reported triggers for migraine attacks in the studied months were stress, lack of sleep, neck pain and anxiety. Table 3 shows the most commonly reported triggers per month and Fig. 1 illustrates the comparison of different years for the four commonest triggers in different months.

The survey questions were answered by 127-1689 users from English speaking countries. Table 4 shows the result of the survey. Out of 1687 users, 55.54\% reported fewer face-to-face specialist's consultations since the beginning of the pandemic but $16.66 \%$ of the users, did not report any change in the number of face-to-face consultations. For telehealth consultations, about $34 \%$ of users reported an increase. Smaller proportions of patients reported less and a similar number of telehealth consultations. $54.29 \%$ and $11.90 \%$ of 1689 respondents preferred face-to-face and telehealth consultation respectively, to communicate with their migraine specialist. The question on the impact of the mode of consultation on migraine outcome was answered by 127 users and $68.80 \%$ of the respondents reported no impact on the frequency of their headaches, with $26.40 \%$ experiencing more and $4.80 \%$ experiencing less migraine attacks. Of the 1565 respondents, most reported $(60.83 \%)$ that telehealth had no effect on their quality of care but $20.64 \%$ reported that telehealth had a negative impact.

\section{Discussion and conclusion}

The frequency of migraine increased in 2020 compared to similar periods of 2019 and 2018. In addition, an overall trend of increase was seen in migraine frequency from 2018 to 2020. A mild reduction in the percentage of the four most reported headache attack triggers, stress, lack of sleep, neck pain and anxiety, was reported in 2020 compared to previous years. The reduction was higher for stress and lack of sleep. Furthermore, patients reported a change in the mode 
Table 1 Basic characteristics of the users enrolled in the study

\begin{tabular}{|c|c|c|c|c|c|c|c|c|c|c|c|c|}
\hline & \multicolumn{4}{|l|}{2018} & \multicolumn{4}{|l|}{2019} & \multicolumn{4}{|l|}{2020} \\
\hline & Jan & Feb & Mar & Apr & Jan & $\mathrm{Feb}$ & Mar & Apr & Jan & Feb & Mar & Apr \\
\hline Total (number) & 120,827 & 109,791 & 113,470 & 109,529 & 124,781 & 125,164 & 129,999 & 127,140 & 151,724 & 141,523 & 123,989 & 118,669 \\
\hline \multicolumn{13}{|l|}{ Gender (\%) } \\
\hline Female & 69.1 & 68.5 & 69 & 68.7 & 61.6 & 61.3 & 61.6 & 61.4 & 56.2 & 58 & 60.7 & 61.3 \\
\hline Male & 6.6 & 7.0 & 7 & 7.5 & 7.2 & 6.9 & 7 & 7 & 8 & 8.4 & 8.8 & 8.6 \\
\hline Unknown & 24.3 & 24.5 & 24 & 23.8 & 31.2 & 31.8 & 31.4 & 31.6 & 35.8 & 33.6 & 30.5 & 30.1 \\
\hline \multicolumn{13}{|l|}{ Age (\%) } \\
\hline$<18$ years & 0.7 & 0.8 & 0.8 & 0.9 & 0.9 & 0.9 & 0.9 & 0.9 & 1 & 1 & 1.1 & 1 \\
\hline $18-54$ years & 39.3 & 38 & 37.6 & 36.3 & 32.3 & 32.1 & 31.6 & 31 & 19.7 & 20 & 21.1 & 21.6 \\
\hline$>55$ years & 1.7 & 1.8 & 1.8 & 1.8 & 1.8 & 1.8 & 1.8 & 1.8 & 1.5 & 1.6 & 1.6 & 1.6 \\
\hline Unknown & 58.3 & 59.4 & 59.8 & 61 & 65 & 65.2 & 65 & 66.3 & 77.8 & 77.4 & 76.2 & 75.8 \\
\hline \multicolumn{13}{|l|}{ Country (\%) } \\
\hline Australia & 1.9 & 1.9 & 2.2 & 2.4 & 2 & 2 & 2.3 & 2.3 & 1.9 & 2 & 2.1 & 2.2 \\
\hline Canada & 4.9 & 5.5 & 6.3 & 6.3 & 5.7 & 5.8 & 6 & 6 & 5.1 & 5.2 & 5.5 & 5.7 \\
\hline France & 7 & 5.9 & 7.2 & 7.1 & 9.6 & 8.7 & 8 & 7.4 & 5.9 & 6.1 & 5.4 & 5.5 \\
\hline Germany & 4.7 & 4.4 & 4.5 & 4.3 & 4.1 & 4.2 & 4.2 & 4.5 & 4 & 4 & 4.1 & 4.4 \\
\hline Italy & 2.7 & 2.5 & 2.4 & 2.1 & 1.8 & 2.9 & 3.2 & 2.9 & 3.2 & 3 & 2.4 & 2.4 \\
\hline Japan & 3.4 & 3.7 & 3.9 & 4.1 & 3.8 & 3.8 & 3.6 & 3.6 & 3.7 & 3.9 & 4.6 & 4.7 \\
\hline Spain & 3.3 & 3.2 & 2.8 & 2.7 & 2.7 & 3.3 & 3.1 & 3.4 & 3.7 & 3.5 & 3.1 & 3.1 \\
\hline Switzerland & 0.9 & 0.8 & 0.8 & 0.8 & 0.7 & 0.7 & 0.7 & 0.7 & 0.8 & 0.8 & 0.8 & 0.8 \\
\hline United Kingdom & 7.5 & 7.1 & 7.1 & 7.2 & 6.7 & 7 & 7.1 & 6.7 & 7.2 & 6.9 & 6.7 & 7 \\
\hline United States & 45.9 & 46.5 & 45.6 & 46.8 & 48 & 47.2 & 47.3 & 47.2 & 46.9 & 47.3 & 47.9 & 46.8 \\
\hline Other & 17.6 & 18.3 & 16.9 & 16 & 14.7 & 14.2 & 14.3 & 15.1 & 17.4 & 17.1 & 17.2 & 17.3 \\
\hline Unknown & 0.2 & 0.2 & 0.3 & 0.2 & 0.2 & 0.2 & 0.2 & 0.2 & 0.2 & 0.2 & 0.2 & 0.1 \\
\hline
\end{tabular}

Table 2 Mean $( \pm S D)$ headache frequency per month

\begin{tabular}{llll}
\hline & \multicolumn{3}{l}{ Year } \\
\cline { 2 - 4 } & 2018 & 2019 & 2020 \\
\hline January & $4.82 \pm 4.95$ & $5.30 \pm 5.38$ & $5.34 \pm 5.41$ \\
February & $4.69 \pm 4.72$ & $5.01 \pm 4.99$ & $5.27 \pm 5.2$ \\
March & $4.99 \pm 5.11$ & $5.35 \pm 5.43$ & $5.51 \pm 5.49$ \\
April & $5.01 \pm 5.06$ & $5.27 \pm 5.31$ & $5.44 \pm 5.34$ \\
Total & $4.88 \pm 4.96$ & $5.23 \pm 5.28$ & $5.39 \pm 5.36$ \\
\hline
\end{tabular}

Comparison of 2018 versus 2019, 2019 vs. 2020 and 2018 versus 2020 for all months were statistically significant $(p<0.001)$ except for the Jan 2019 vs. Jan 2020 comparison $(p=0.052)$

of specialist's consultation during the pandemic, but this had no impact on patient's quality of migraine management.

Our findings have been previously studied in the literature.

\section{Headache frequency}

We showed an increase in the frequency of headache in patients experiencing migraine during the pandemic compared to similar periods in the previous two years. Similar results were found in patients from Northern Italy [7], and the authors hypothesised that there could be a relationship between the severity of the COVID-19 pandemic in this region of Italy and an increase in the frequency and intensity of headache. Al-Hashel et al. [8] in 1018 patients from Kuwait also showed that about $59.5 \%$ of participants experienced an increase in the frequency of migraine during the pandemic. They reported an association between poor migraine outcomes and female sex, short duration of migraine disease, difficulties accessing neurologist visits, working in time of pandemic and difficulties accessing migraine medications.

However, Delussi et al. [7] found a decrease in the frequency and intensity of headache in 433 migraine patients from Italy. They reported a relationship between this finding and the number of stay-at-home days. Another study from Italy [9] including 147 migraine patients treated with anti-calcitonin gene related peptide (CGRP) monoclonal antibodies (erenumab or galcanezumab) showed a reduction in headache days per month and headache impact test score in the first lockdown month compare to the prior month. This effect plateaued with time but after 3 months, there was still a mild reduction in headache frequency. 
Table 3 Most reported triggers in each month

\begin{tabular}{|c|c|c|c|c|c|c|c|c|c|c|c|c|}
\hline & \multicolumn{4}{|l|}{2018} & \multicolumn{4}{|l|}{2019} & \multicolumn{4}{|l|}{2020} \\
\hline & Jan & Feb & Mar & Apr & Jan & Feb & Mar & Apr & Jan & Feb & Mar & Apr \\
\hline Number of users & 120,827 & 109,791 & 113,470 & 109,529 & 124,781 & 125,164 & 129,999 & 127,140 & 151,724 & 141,523 & 123,989 & 118,669 \\
\hline \multicolumn{13}{|l|}{ Triggers (\%) } \\
\hline Stress & 39.7 & 39.1 & 40.4 & 39.5 & 37.9 & 37.7 & 39.3 & 38.9 & 35.9 & 35.6 & 38.4 & 34.4 \\
\hline Lack of sleep & 25.5 & 24.1 & 25.3 & 25.3 & 25.4 & 23.7 & 25.7 & 25.1 & 24.3 & 23.1 & 22.7 & 21.2 \\
\hline Neck pain & 20.3 & 15.6 & 20 & 20.3 & 20.7 & 19.9 & 20.8 & 20.3 & 19.8 & 19.3 & 19.3 & 18.9 \\
\hline Anxiety & 19.0 & 18.5 & 19.1 & 19.2 & 18.9 & 17.8 & 18.7 & 18.3 & 17.1 & 17.1 & 20.7 & 18.5 \\
\hline Interrupted sleep & 17.4 & - & - & - & - & - & - & - & 17.5 & - & - & - \\
\hline Very variable weather & - & 17.7 & 17.3 & 19.4 & 18.4 & 19.9 & 18.7 & 20.3 & - & 18.1 & 18.3 & 19.1 \\
\hline
\end{tabular}

Users may have selected more than one choice

January

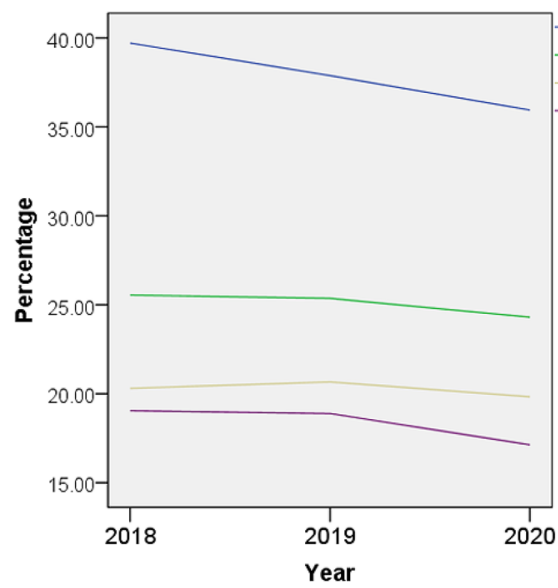

March

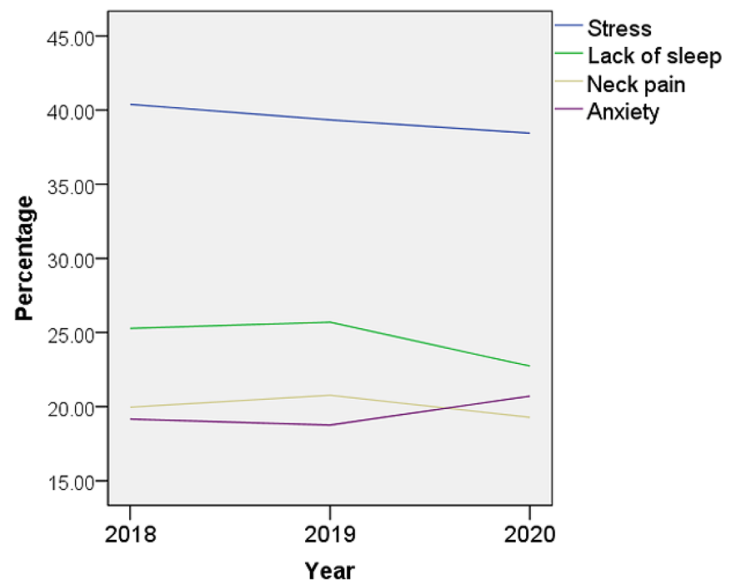

February

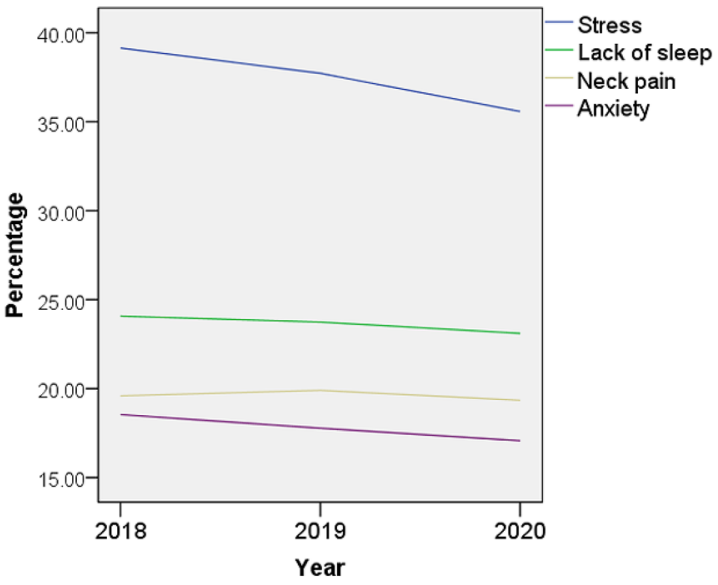

April

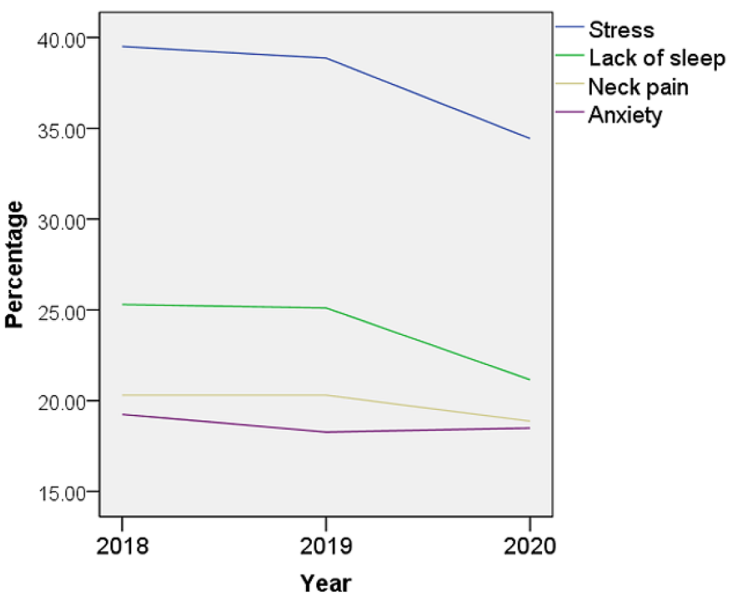

Fig. 1 Comparison of three consecutive years for the four most reported triggers in each month. Two triggers (interrupted sleep and very variable weather) were not common in different months and are excluded from the figures 
Table 4 Responses to the survey of COVID-19 impact on migraine management, face-to-face or telehealth consultations

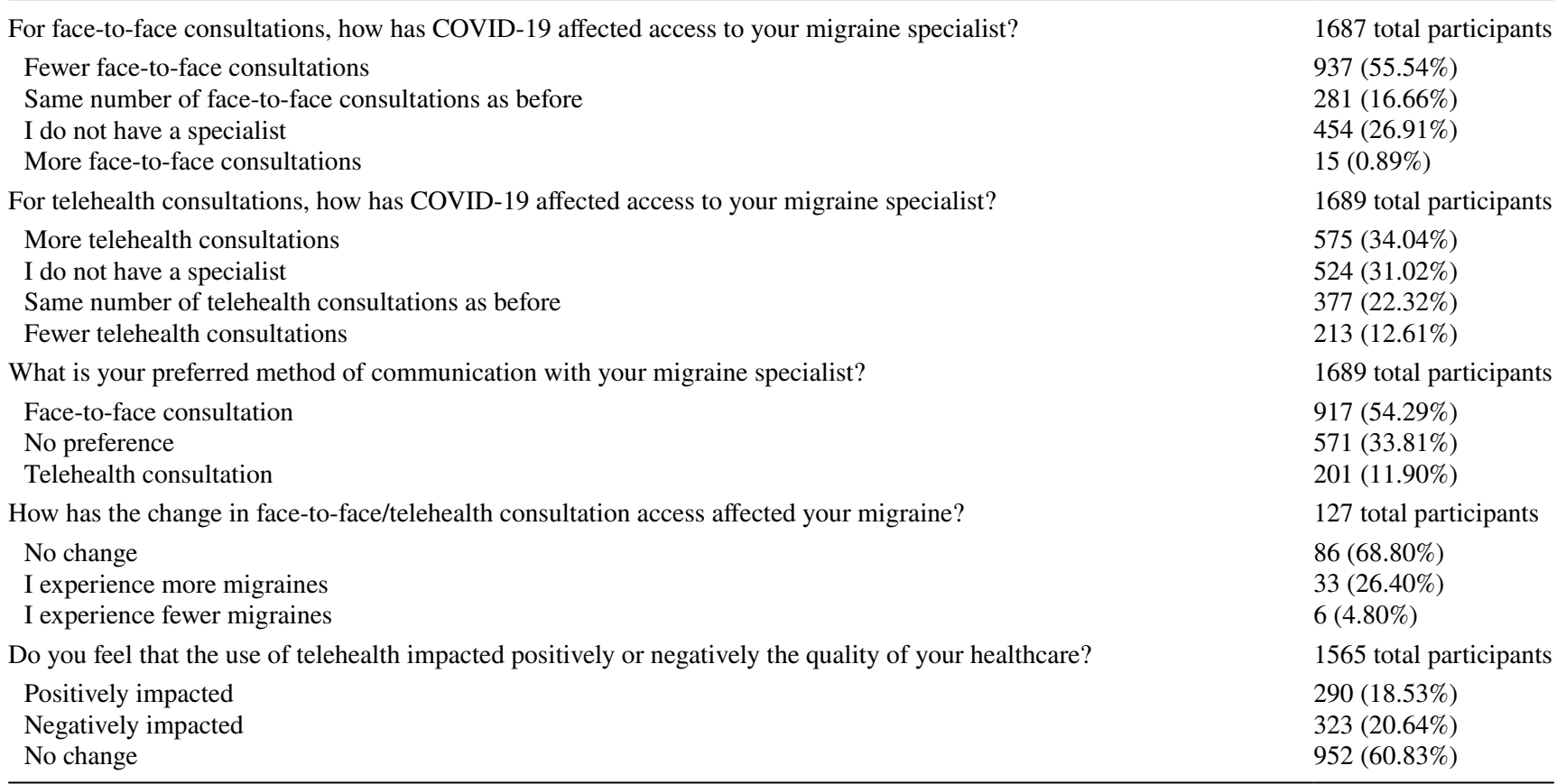

Parodi et al. [10] compared the first 2 months of quarantine with the 2 months prior to the lockdown in Italy and showed that migraine patients had less migraine attacks and less severe headaches during the quarantine. Similarly, another study in Italy by Dallavalle et al. [11] on children and adolescents in Northern Italy showed a decrease in the frequency and intensity of migraine, with and without aura, following the lockdown. Papetti et al. [12] showed the same results and suggested that the decrease in school effort and anxiety were associated with a reduction in the frequency and intensity of headaches. However, longer headache disease duration was associated with lower decrease in headache frequency and intensity. Similar to these reports, another study from the Netherlands showed a decrease in migraine frequency and an increase in general well-being in migraine patients during the lock down compared to a month before the lock down [13].

\section{Migraine triggers and comorbidities}

We showed that migraine triggers including stress, lack of sleep, neck pain and anxiety decreased to a small extent during the pandemic. There were no published papers looking at migraine triggers in the time of the pandemic. However, anxiety, mood changes and increase in psychological distress associated with higher frequency of attacks and attention paid to media about COVID-19 has been reported as comorbidities in patients with migraine [7, $11,14]$.

\section{Migraine management}

In the survey of patients with migraine, we showed that since the pandemic, interactions with specialists had changed with more telehealth and fewer face-to-face visits. Patients, however, preferred face-to-face to telehealth but did not report a change in the quality of care between both methods. Similarly, telehealth was found to be effective and safe compared to traditional consultations methods in non-acute headaches and there was no difference in intensity and headache impact between the methods [15]. This was confirmed in a study conducted in India where telehealth was found to be feasible and as effective as face-to-face consultations in children with migraine [16].

\section{Strengths and limitations}

A key strength of our study is the use of a large sample of migraine-specific phone application users (over 100,000 users), which more robustly reflect the real-life experience. In our analysis, data were obtained from users from different countries, age and genders. We used rigorous statistical techniques to analyse and present the data.

$\mathrm{We}$, however, recognise that there are some limitations. First, data in the current study are self-reported. It might be that the increase in the frequency of headache is caused by a change in reporting habits (for example due to more free time during the pandemic and increased confidence in using the application) with more assiduous use of the Migraine Buddy phone application during the pandemic. However, no 
additional reminders about completing data in the application were sent to users, compared to previous years. Moreover, the high number of users should dilute this possible bias effect. In addition, it is important to mention another limitation for this self-reported data. One of the triggers that was reported in the phone application was neck pain which can be a migraine symptom and even a prodromal phenomenon [17] and not a trigger and reported data cannot distinguish them.

Adjustment for confounding factors associated with the change in frequency was not possible due to the lack of data on these attributes, given the retrospective nature of this analysis. As the pandemic began at different times of the year in each country, with differences in peak infections, comparisons between countries could give a better understanding of the pattern which is not included in the current paper. In addition, this difference in peak time of the pandemic from country to country with the selected time period in our study may be a reason for the different result of the current paper with some previous reports. Furthermore, data on other triggers could help the understanding of the changes in the four most mentioned triggers. Finally, the COVID-19 infection may have an impact on migraine disease. Our study lacks data about the migraine changes in infected migraine patients which have been reported in other studies [8] [7] [18].

\section{Summary and conclusion}

In our large study, we showed that the frequency of migraine attacks increased during the COVID-19 pandemic. The increase in the frequency of migraine headache in 2020 compared to the same period in 2019 and 2018 likely represents a real increase but we were unable to ascertain the reason for this change and it can be due to more use of the application by the users. One other finding from our study was that the four most reported headache triggers were stress, lack of sleep, neck pain and anxiety. These, however, cannot explain the increase in frequency as stress and lack of sleep especially decreased during the pandemic. This is possible that other triggers such as staying at home, having to adjust to working from home, home schooling requirements or being unwell with COVID-19 has led to the increase in the frequency of migraine. Importantly, Migraine Buddy users reported a preference for face-to-face consultation over telehealth but this had no impact on the quality of care they perceived.

In conclusion, the frequency of migraine has increased during the COVID-19 pandemic but this is not explained by changes in the most common triggers such as stress and lack of sleep. Specialists' consultations via telehealth are feasible, acceptable and safe for the management of patients experiencing migraine.

\section{Unanswered questions and suggestions for further targeted research}

This is a cross-sectional study and further investigations are required to understand the patterns reported in the study. Comparison between countries, longer study duration, and inclusion of potential triggers might lead to a better understanding of the trends. Furthermore, classifying the users based on the change in the percentage of the most reported triggers (increased vs. decreased) and comparing them could help identifying factors associated with these triggers.

In addition, selecting random samples from the application users in different countries, performing survey studies and checking for different variables such as migraine duration and medication status, having a stable or an unstable disease condition, recording number of stay-at-home days, comorbidities and job status may give more information about the effect of COVID-19 pandemic on migraine disease.

\section{Clinical, scientific and/or public health implications}

1- Migraine, a prevalent and chronic condition, is impacted by the COVID-19 pandemic. In general, an increase in the frequency of headache is reported in the Migraine Buddy phone application during the pandemic compared to before. This can be a real increase or just the result of more use of the application by the users. However, health care professionals and caregivers should pay more attention to migraine patients during the pandemics and lockdown.

2- The four most reported triggers of migraine headache attacks in the Migraine Buddy phone application are stress, lack of sleep, neck pain and anxiety. These factors must be taken into consideration when interacting with migraine patients.

3- A low-level reduction in the percentage of the four most reported triggers is reported by Migraine Buddy users.

4- Migraine patients had fewer face-to-face and more telehealth consultations during the pandemic compared to before. Most patients preferred face-to-face to telehealth consultations. However, in most users' opinion, the method of interaction with their specialist did not have on impact on their quality of care.

Acknowledgements Data are provided by employees of Healint Pte Ltd. The authors would like to thank Weijie Poh, PhD, and François Cadiou for their collaboration and for providing the data. 
Author contributions All the authors contributed to the study design, data analysis and drafting the manuscript. DC, Employee of Healint Pte Ltd., provided the data. The manuscript is read and approved by all the authors.

Funding No funding required.

Data availability (Data transparency) De-identified data used in this analysis will be shared by request from any qualified investigator via the Research Office of The George Institute for Global Health.

\section{Declarations}

Conflicts of interest FH, CC, AR and CD declare no conflict of interest. DC is Employee of Healint Pte Ltd. Data are provided by the employees of Healint Pte Ltd. (owner of the Migraine Buddy application). Results of the study were presented in Migraine Trust Virtual Symposium 2020 (MTIS2020), 3-9 October 2020.

Ethical approval Data reported by users of the Migraine Buddy Phone application were used for this secondary data analysis. The study does not need ethical approval.

Consent to participate User data were deidentified and users gave permission that their data can be used for research purposes.

\section{References}

1. Coronavirus Updates. Last updated: September 13, 2020, 06:12 GMT. (2020). https://www.worldometers.info/coronavirus/.

2. Global, regional, and national incidence, prevalence, and years lived with disability for 328 diseases and injuries for 195 countries, 1990-2016: a systematic analysis for the Global Burden of Disease Study 2016 (2017). Lancet. 390:1211-1259. https://doi. org/10.1016/s0140-6736(17)32154-2

3. Chen PM, Hemmen TM (2020) Evolving healthcare delivery in neurology during the Coronavirus Disease 2019 (COVID-19) pandemic. Front Neurol 11:578. https://doi.org/10.3389/fneur.2020. 00578

4. Szperka CL, Ailani J, Barmherzig R, Klein BC, Minen MT, Halker Singh RB, Shapiro RE (2020) Migraine care in the era of COVID-19: clinical pearls and plea to insurers. Headache 60(5):833-842. https://doi.org/10.1111/head.13810

5. World Health Organization (2020) The impact of the COVID-19 pandemic on noncommunicable disease resources and services: results of a rapid assessment.

6. MedCalc -easy-to-use statistical software. https://www.medcalc. org/calc/comparison_of_means.php.

7. Delussi M, Gentile E, Coppola G, Prudenzano AMP, Rainero I, Sances G, Abagnale C, Caponnetto V, De Cesaris F, Frattale I, Guaschino E, Marcinnò A, Ornello R, Pistoia F, Putortì A, Roca ME, Roveta F, Lupi C, Trojano M, Pierelli F, Geppetti P, Sacco S, de Tommaso M (2020) Investigating the effects of COVID-19 quarantine in migraine: an observational cross-sectional study from the Italian National Headache Registry (RICe). Front Neurol 11:597881. https://doi.org/10.3389/fneur.2020.597881

8. Al-Hashel JY, Ismail II (2020) Impact of coronavirus disease 2019 (COVID-19) pandemic on patients with migraine: a web-based survey study. J Headache Pain 21(1):115. https://doi.org/10.1186/ s10194-020-01183-6

9. Altamura C, Cevoli S, Aurilia C, Egeo G, Fofi L, Torelli P, Brunelli N, Pierangeli G, Favoni V, Fallacara A, Pensato U, Barbanti P, Vernieri F (2020) Locking down the CGRP pathway during the COVID-19 pandemic lockdown: the PandeMig study. Neurol Sci 41(12):3385-3389. https://doi.org/10.1007/ s10072-020-04767-x

10. Parodi IC, Poeta MG, Assini A, Schirinzi E, Del Sette P (2020) Impact of quarantine due to COVID infection on migraine: a survey in Genova. Italy Neurol Sci 41(8):2025-2027. https://doi.org/ 10.1007/s10072-020-04543-x

11. Dallavalle G, Pezzotti E, Provenzi L, Toni F, Carpani A, Borgatti $\mathrm{R}$ (2020) Migraine symptoms improvement during the COVID-19 lockdown in a cohort of children and adolescents. Front Neurol 11:579047. https://doi.org/10.3389/fneur.2020.579047

12. Papetti L, Loro PAD, Tarantino S, Grazzi L, Guidetti V, Parisi P, Raieli V, Sciruicchio V, Termine C, Toldo I, Tozzi E, Verdecchia P, Carotenuto M, Battisti M, Celi A, D'Agnano D, Faedda N, Ferilli MA, Grillo G, Natalucci G, Onofri A, Pelizza MF, Ursitti F, Vasta M, Velardi M, Balestri M, Moavero R, Vigevano F, Valeriani M (2020) I stay at home with headache. A survey to investigate how the lockdown for COVID-19 impacted on headache in Italian children. Cephalalgia 40(13):1459-1473. https://doi.org/10.1177/ 0333102420965139

13. Verhagen IE, van Casteren DS, Lentsch SV, Terwindt GM (2021) Effect of lockdown during COVID-19 on migraine: A longitudinal cohort study. Cephalalgia:333102420981739. https://doi.org/10. 1177/0333102420981739

14. Ma M, Fang J, Li C, Bao J, Zhang Y, Chen N, Guo J, He L (2020) The status and high risk factors of severe psychological distress in migraine patients during nCOV-2019 outbreak in Southwest China: a cross-sectional study. J Headache Pain 21(1):100. https:// doi.org/10.1186/s10194-020-01168-5

15. Müller KI, Alstadhaug KB, Bekkelund SI (2017) A randomized trial of telemedicine efficacy and safety for nonacute headaches. Neurology 89(2):153-162. https://doi.org/10.1212/wnl.00000 00000004085

16. Sharawat IK, Panda PK (2020) Caregiver satisfaction and effectiveness of teleconsultation in children and adolescents with Migraine during the ongoing COVID-19 pandemic. J Child Neurol: 883073820968653. https://doi.org/10.1177/0883073820 968653

17. Lampl C, Rudolph M, Deligianni CI, Mitsikostas DD (2015) Neck pain in episodic migraine: premonitory symptom or part of the attack? J Headache Pain 16:566. https://doi.org/10.1186/ s10194-015-0566-9

18. Membrilla JA, de Lorenzo Í, Sastre M, Díaz de Terán J (2020) Headache as a cardinal symptom of Coronavirus Disease 2019: a cross-sectional study. Headache 60:2176-2191. https://doi.org/ 10.1111/head.13967 